\title{
School lunch and learning behaviour in secondary schools: an intervention study
}

\author{
C. Storey ${ }^{1}$, J. Pearce ${ }^{1}$, P. Ashfield-Watt ${ }^{1}$, L. Wood ${ }^{1}$, E. Baines ${ }^{2}$ and M. Nelson ${ }^{1}$ \\ ${ }^{1}$ School Food Trust, Moorfoot, Sheffield S1 4PQ, UK and ${ }^{2}$ Psychology and Human Development, Institute of Education, \\ 25 Woburn Square, London WC1H OAA, UK
}

The belief that children who are well fed are better learners is supported by observational evidence from developing countries, but there is a dearth of good-quality research on the effect of diet on learning in school-aged children in developed countries ${ }^{(1)}$. The current study helped to redress this by investigating the effect of catering and dining interventions in secondary schools on classroom behaviour in the post-lunch period using a validated direct observational method.

In 2008, all the co-educational secondary schools across four Local Authority (LA) areas in England were approached to participate in a school lunch and behaviour study. Three schools were identified in each LA. Within each triplet, two schools were randomly allocated to receive the intervention and one school acted as a control. A food and dining room checklist was used to record nutritional and environmental characteristics at each school and to generate a list of suggested interventions. Intervention schools received £2000 towards making the planned changes.

Food interventions included a range of activities: improving the compliance of school lunch menus with the food-based standards; healthy eating workshops, marketing activities; fruit and vegetable packs available at lunchtime, etc. Dining room interventions involved temporal and physical changes: staggering lunch times; modifying the layout and queuing system; redecorating, displaying students' artwork and buying new furniture. Control schools did not implement any study-specific interventions, but were offered the intervention advice and the financial support at the end of the 15 -week study period.

At each school, seven pupils were randomly selected from year groups 7 and 9. Each pupil was observed in post-lunchtime classes across a range of disciplines. Trained observers used a protocol modified from Blatchford et al. ${ }^{(2)}$. Pupils were considered to be 'on-task' (concentrating and alert) or 'off-task' (disruptive or disengaged) depending on whether their social mode of engagement (working alone, working with other pupils, interacting with the teacher) was appropriate for the type of work setting they were expected to be engaging in. The observers were not aware of the school's status (control or intervention). A total of 156 pupils had their behaviours observed (control, $n$ 58, intervention, $n$ 98) at baseline (12210 and 20560 observations, control and intervention, respectively) and at follow-up (16846 and 23462 , respectively).

On-task and off-task behaviours were similar across treatment groups at baseline. At follow-up, on-task behaviour had increased and off-task behaviour had decreased in both groups, but the changes were significantly greater in the intervention group. Intervention group pupils were $18 \%$ more likely to be on-task (OR $1.18,95 \%$ CI $1.05,1.33$ ) and $14 \%$ less likely to be off-task (OR $0.86,95 \%$ CI $0.75,0.98)$ compared with control group pupils at the end of the intervention period.

This study shows that modifying food provision and dining environment can improve learning-related behaviours of secondary school pupils in the post-lunch period. This finding supports on-going investment and interventions by local authorities across the UK to improve school food and lunchtime dining facilities.

1. Ells LJ, Hillier FC, Shucksmith J et al. (2008). A systematic review of the effect of dietary exposure that could be achieved through normal dietary intake on learning and performance of school-aged children of relevance to UK schools. Br J Nutr 100, 927-936.

2. Blatchford P, Bassett P \& Brown P (2005) Teachers' and pupils' behaviour in large and small classes: a systematic observation study of pupils aged 10/11 years. J Educ Psychol 97, 3, 454-467. 